\title{
Programa Emprendedor Obligatorio. Alternativa de Educación Integral
}

\author{
E.A. Chapa \\ Instituto Politécnico Nacional, E.S.I.Q.I.E. Ciencias Básicas, Av. I.P.N. 2126 De. 7F - 501, \\ Col. San José de Ticomán, 07340 México, D.F-México
}

\section{Resumen}

Este artículo discute la aplicación de un programa emprendedor para las carreras de ingeniería en la educación superior. El sistema de acreditación en México, el cual se aplica a esas escuelas, considera la inclusión de algunos cursos en ciencias sociales, pero el número de cursos apropiados para la formación integral de los futuros ingenieros es limitada. Por eso, se propone y analiza un programa emprendedor el cual incluye cursos tales como finanzas, marketing, relaciones humanas y sicología social. El programa muestra ser efectivo, ya que expone a los estudiantes a casos prácticos con los cuales se encontrarán durante su vida profesional.

Palabras claves: programa emprendedor, currículo, educación integral, educación en ingeniería

\section{Compulsory Enterprising Program. An Alternative Path for Integral Education}

\begin{abstract}
This article discusses the application of an enterprising program for engineering careers in higher education schools. The accreditation system in Mexico, which applies to these schools, considers the inclusion of some courses in social sciences, but the number of courses needed for the integral formation of the future engineers is limited. Therefore, an enterprising program which includes courses such as finances, marketing, human relations and social psychology is proposed and analyzed. The program has shown to be effective since it exposes students to practical cases that they will find during their professional life.
\end{abstract}

Keywords: enterprising program, curricula, integral education, engineering education

Nota: este artículo está tomado de "Información Tecnológica" [ISSN 0716-8756], vol. 9(2), 353-357 (1998) 


\section{INTRODUCCION}

Es conocido al revisar los currículum de las carreras de Ingeniería Química actuales, el limitado número de asignaturas de las áreas sociales y económico-administrativas como: Finanzas, Mercadotecnia, Relaciones Humanas, Psicología Social, etc., y la necesidad de ellas con la finalidad de poder acreditar y certificar los planes y programas de estudio, con motivo de la entrada del tratado de libre comercio a México.

Por esta causa, la necesidad de implementación de dichas asignaturas, lleva a proponer en las instituciones públicas de educación superior, el programa de "jóvenes emprendedores", el cual incluye asignaturas de las áreas mencionadas, con el fin de lograr una educación integral en los educandos, así como actividades orientadas al fomento de la creatividad de los jóvenes mediante el lema "emprende, aprendiendo".

\section{METODOLOGÍA}

En principio, ante ésta revisión de planes y programas de estudio, se propone como metodología:

1. La inserción en la tira de materias el programa de jóvenes emprendedores, que ofrezca posibilidad de conocer y entender de manera práctica el sistema de economía de mercado con contenido social, desarrollándoles educativamente el espíritu emprendedor.

2. Hacer que se conozca de manera práctica, los principios básicos de operación de una empresa: sus fines y condiciones de creación, los tipos de actividades que se realizan en ellas, sus recursos y las obligaciones que tiene.

3. Hacer que se comprendan las relaciones de una empresa con los demás elementos de una economía de mercado.

4. Valorar las actitudes que caracterizan el espíritu emprendedor: iniciativa, búsqueda de superación, creatividad y disposición para el trabajo en equipo.

Para una carrera de ingeniería química se proponen la creación de empresas de la siguiente manera: Cuando se ha pasado por el área del conocimiento básico, y se lleva algo avanzado en el área de fundamentos de ingeniería, de acuerdo al nuevo mapa curricular, (en términos reales el quinto semestre), insertar durante el quinto y sexto semestre, como obligatoria la creación de una empresa juvenil. Cada empresa juvenil se crea con 10 estudiantes, en la que cada uno de ellos juega un rol particular. Así, asesorados por expertos en cada una de las áreas se siguen los siguientes pasos:

1. Se decide en principio, el tipo de producto a fabricar y/o distribuir, o el tipo de servicio a ofrecer, ya que estas empresas pueden ser de productos o de servicios.

2. Se realiza un estudio de mercado, del producto o servicio seleccionado.

3. Se obtienen los costos de producción y operación.

4. Se empieza con un capital social, acorde con los costos previstos.

5. Se nombran los responsables de cada área como son: Director General, Gerencia de Producción, Gerencia de Mercadotecnia, Gerencia de Recursos Humanos, Gerencia de Control de Calidad y Gerencia de Finanzas. Los demás integrantes de la empresa serán los responsables de las ventas, sin que con ello quiera decir que los demás integrantes de la empresa, en su momento también se convierten en vendedores.

6. Se venden las acciones de la empresa, entre amigos, familia, etc. 
7. Se empieza el proceso de producción con todas las implicaciones que tiene, hasta llegar al producto terminado.

8. Conforme se empiezan a sacar los lotes de productos terminados, se empezará con la etapa de mercadotecnia y así sucesivamente.

9. El programa consta de 32 semanas, en las cuales se distribuyen las diferentes tareas a realizar.

10. En el transcurso de las 32 semanas, se ofrecen cursos obligatorios a los responsables de las diferentes áreas.

11. A partir de la semana 28 , se empieza con el proceso de liquidación de la empresa, con el fin de aprender todo lo inherente a ello. Si la empresa ha tenido utilidades, se paga a los accionistas las mismas.

12. Es importante contar con alguna cantidad estipulada para la operación de la empresa, pues esto funciona únicamente las 32 semanas.

13. Se hace el cierre de la empresa, si esta funciona de una manera adecuada, los integrantes de la misma podrán decidir a abrir una empresa de tales características, ya de manera formal y no únicamente con el objetivo de emprender aprendiendo.

\section{RESULTADOS y DISUCUSIÓN}

El proyecto de modernización económica y su complemento indispensable - la reforma de las instituciones políticas y sociales - hacen de la educación un área de interés fundamental para la transformación estructural que México enfrenta hoy. Más importante aún, el mejoramiento cualitativo de la educación permitirá el ingreso de un mayor número de mexicanos a los nuevos procesos productivos, a partir de lo cual será posible lograr una distribución más equitativa de los beneficios emanados del crecimiento económico.

La educación "como precondición del desarrollo no puede disociarse de lo político y de lo social. Es indispensable reconocer la dificultad de propugnar y cristalizar reformas institucionales en una sociedad que carece de niveles escolares satisfactorios" (Rubio, 1992). Una educación fragmentaria, inequitativa, parcial y de baja calidad no estimula ni facilita la participación social, ya que ésta es la que, en definitiva, determina el carácter y la dinámica de las sociedades modernas y democráticas: no es posible concebir una sociedad que por un lado reproduzca eficazmente las condiciones propicias de la esfera económica, y por el otro, permanezca el margen de las formas de comunicación y de organización que tienen lugar en la esfera política y social. Estas esferas son indisociables en la realidad; el desarrollo no puede reflejarse sino en ambas. Para ello la educación no solo es el mejor insumo, sino el factor indispensable.

Una empresa de jóvenes emprendedores es un grupo que realiza una actividad educativa, la cual facilita conocer como se desarrollan las empresas que operan en el sistema económico. La actividad proporciona una experiencia práctica que reproduce de manera realista muchas de las funciones que realizan esas empresas. El lema a discutir será "Emprender, aprendiendo".

La importancia de la formación de empresas juveniles por parte de los jóvenes de educación superior ayuda también en cuanto a la deserción estudiantil por falta de recursos debido a que "el financiamiento de la educación superior en México ha sido uno de los renglones más afectados por la crisis económicas de los ochentas y noventas, y por el ajuste de las finanzas públicas que se han realizado" (Torrico, 1986)

Las empresas de jóvenes emprendedores redundarán en una fuerza de trabajo con experiencia y capacidades amplias, estos hechos "implican que las habilidades adquiridas en un momento dado por el estudiante lo obligará -se quiere mantenerse empleado- a actualizar continuamente sus habilidades a través de procesos de educación continua" (Chubb y Moe, 1990), la experiencia 
adquirida en las empresas juveniles posibilita a los jóvenes para realizar un grupo variado de tareas de producción y para enfrentarse a problemas inesperados y a tecnologías nuevas.

Los resultados son planteados en función de las empresas juveniles que han participado en forma voluntaria en institución que representó.

Una empresa juvenil dedicada a la fabricación de una crema que inicialmente se pensó para la limpieza de los mecánicos y que después de comprobó que era multiusos, formada por jóvenes estudiantes de $5^{\circ}$ semestre de la carrera de Ingeniería Química, la cual obtuvo el primer lugar entre las empresas de todo el Instituto Politécnico Nacional en presentación del producto, dicha empresa después de haber sido observada por integrantes del Programa Nacional de Solidaridad, fue solicitada al I.P.N. para ser una empresa de Solidaridad, con lo cuál se daría empleo a cientos de trabajadores. Sin embargo por problemas de patente del producto, no fue posible su explotación por parte del programa. Dicha empresa obtuvo utilidades del 31\% en 25 semanas de operación.

Haciendo un seguimiento de los jóvenes integrantes de la empresa, en el momento actual, cuatro de ellos aún sin haber terminado sus estudios profesionales, están colocados en tres empresas en las cuales se realiza tecnología de punta en el país, con muy buenos resultados en su comportamiento social y productivo.

Otra empresa integrada por estudiantes del $3^{\circ}$ semestre de la carrera de Ingeniería Química y dedicada a la fabricación de un producto de limpieza de uso doméstico, obtuvo los primeros lugares en las áreas de mercadotecnia y recursos humanos, habiendo obtenido utilidades del orden del $25 \%$ en 25 semanas de operación.

Al hacer comparaciones en el comportamiento de jóvenes que han integrado alguna empresa juvenil y los que no, los primeros tienen un mejor desenvolvimiento ante los retos que representa la vida, el empleo y la creación de tecnología.

Por todo lo anterior en algunas de las carreras de Ingeniería Química se está planteando en tira de materias como algo obligatorio a partir del $5^{\circ}$ semestre de las mismas. Esto me lleva a proponerlo como una forma de educación integral en la cual, por un lado se relacionan las asignaturas propias de la carrera y por otra las asignaturas de las áreas de Ciencias Sociales y económico-administrativas, necesarias para la acreditación y certificación de nuestros planes y programas de estudio.

La efectividad de la creación de empresas juveniles con el fin de emprender y aprender ha sido un insumo ó -una variable independiente- en el proceso de enseñanza aprendizaje sin embargo las "especificaciones de estos insumos resultan de varios modelos educativos ya experimentados en México y otros países" Blaugh (1973), por lo que los resultados de la creación de estas empresas tienen un mayor impacto en la investigación y en la política educativa.

\section{CONCLUSIONES}

Los jóvenes emprendedores, son capaces de iniciar proyectos importantes como desarrollar una empresa. También tienen la capacidad para llevarlos adelante, realizando el esfuerzo que sea necesario para lograr sus objetivos.

Los beneficios que tiene el manejar en forma curricular las empresas de jóvenes son: impulsar el desarrollo del estado, mejorar la actitud de los jóvenes hacia el trabajo, formar un ánimo emprendedor en los estudiantes, detectar y promover las vocaciones empresariales, favorecer el autoempleo, facilitar la tranquilidad social, encauzar el liderazgo de la juventud, orientar la vocación profesional de los participantes, complementar la educación tradicional de los jóvenes, vincular al sector productivo con el sector educativo, formación de recursos humanos altamente calificados, gestar una cultura empresarial. 
El propósito inmediato es el aprendizaje, usando la estrategia educativa de hacer funcionar al grupo de manera semejante a una empresa, que opere con éxito y produzca una ganancia económica. Adquieren conocimientos sobre las posibilidades de desarrollo personal que ofrece el mundo de las empresas, así como sobre los esfuerzos que han de realizarse en cada caso para aprovecharlas, todo lo cual puede tener después beneficios económicos.

\section{REFERENCIAS}

Blaugh, M. "Educación y el problema del empleo" Ginebra: Organización Internacional del Trabajo, 1973.

Chubb, J.E. y T. Moe; "Politics, Markets and America's Schools", Washington. The Brookings Institution (1990).

Rubio, L.; “Educación para una Economía Competitiva”, $1^{\circ}$ Edición. Editorial Diana, México (1992)

Torrico, H.T.; "Tendencias Actuales de la Educación Superior en el Mundo", $1^{\circ}$ Edición UNAM (1986). 